\title{
Combined Continuous Ethinyl Estradiol/Norethindrone Acetate Does Not Improve Forearm Blood Flow in Postmenopausal Women at Risk for Cardiovascular Events: A Pilot Study
}

\author{
CLAIRE S. DUVERNOY, M.D., PATRICIA A. ROSE, B.A., H. MYRA KIM, Ph.D., \\ CHRISTINE KEHRER, B.S., and ROBERT D. BROOK, M.D.
}

\begin{abstract}
Objective: This study sought to determine whether combined continuous ethinyl estradiol and norethindrone acetate, a postmenopausal hormone therapy (HT) combination designed to have fewer side effects than cyclical therapies and therapies using medroxyprogesterone acetate (MPA), could improve vascular endothelial function in postmenopausal women with risk factors for cardiovascular disease (CVD).

Methods: Eighteen postmenopausal women (mean age $62 \pm 11$ years) participated in a randomized, placebo-controlled, crossover design trial of $10 \mu \mathrm{g}$ estradiol/1 $\mathrm{mg}$ norethindrone acetate given once daily for 3 months, with a 1-month washout period between placebo and active treatment phases. Vascular reactivity was assessed at each phase of the study using high-frequency brachial artery ultrasound in response to flow-mediated hyperemia, cold pressor testing, and sublingual nitroglycerin. Markers of cardiovascular risk, including cholesterol levels, inflammatory markers, fibrinolytic markers, and solubilized adhesion molecules, were also measured at each phase.

Results: We found no significant difference in vascular reactivity measurements during active treatment with ethinyl estradiol/norethindrone acetate vs. placebo. C-reactive protein (CRP) levels increased significantly during active treatment, and high-density lipoprotein (HDL) levels decreased significantly. Vascular cell adhesion molecule-1 (VCAM-1) levels declined during active treatment. Plasminogen activator inhibitor-1 (PAI-1) levels were inversely correlated with flow-mediated hyperemic vascular reactivity, independent of active treatment or placebo phases.

Conclusions: In this older postmenopausal population with at least one cardiovascular risk factor, treatment with combined continuous ethinyl estradiol and norethindrone acetate failed to improve vascular endothelial function. The agent's proinflammatory effect or subclinical atherosclerosis in this population may have contributed to this finding.
\end{abstract}

Division of Cardiovascular Medicine, University of Michigan, Ann Arbor, Michigan.

This project was sponsored by an unrestricted educational grant from Pfizer, Inc. 


\section{INTRODUCTION}

E STROGEN ADMINISTRATION has been shown by some investigators to improve endotheliumdependent vasodilation in both the peripheral and coronary circulation of postmenopausal women. ${ }^{1,2}$ In spite of these beneficial effects, postmenopausal hormone therapy (HT) using conjugated equine estrogen with medroxyprogesterone acetate (MPA) has not definitively demonstrated beneficial cardiovascular outcomes; indeed, two large randomized trials showed a slightly increased risk of cardiovascular events in women randomized to combined HT. ${ }^{3,4}$ Interest remains in teasing out whether alternative estrogen formulations, different time courses of administration, or selected subpopulations of postmenopausal women may benefit from HT nonetheless. It has been postulated that the use of MPA was responsible for at least some of the adverse effects seen in randomized trials, as MPA has been shown to diminish beneficial estrogen effects on the endothelium in animal studies..$^{5}$ The objective of this study was to determine whether combined continuous ethinyl estradiol and norethindrone acetate, a postmenopausal HT combination designed to have fewer side effects (such as breakthrough bleeding, bloating, and breast tenderness) than therapies using MPA or than cyclical therapies, could improve vascular endothelial function.

Flow-mediated, endothelium-dependent vasodilation of the brachial artery has been shown to correlate with endothelium-dependent coronary vasoreactivity as assessed invasively following intracoronary acetylcholine infusion. ${ }^{6}$ Furthermore, evaluation of brachial artery vasoreactivity is a simple, relatively inexpensive method that is quick to perform and is highly reproducible in the hands of an experienced operator. Several different provocative stimuli may be used to assess the integrity of the vascular endothelium, including reactive hyperemia, mental stress, exercise, and cold pressor testing. The cold pressor test (hand and forearm immersed in ice) is a noninvasive sympathetic stimulus that may be used as a provocative test to assess coronary as well as peripheral vascular endothelium in an analogous fashion to intracoronary acetylcholine infusion. Normal vascular endothelium demonstrates a vasodilator response to epinephrine (a mixed alpha-adrenergic and beta-adrenergic ag- onist), which can be reversed to a vasoconstrictor response after removal of the endothelium. Zeiher et al. ${ }^{7}$ demonstrated that coronary artery responses to cold pressor testing exactly paralleled the response to acetylcholine. Patients with normal coronaries showed vasodilation in response to cold and after acetylcholine infusion, and those with minor irregularities of coronary vasculature and those with documented coronary stenoses showed paradoxical vasoconstriction. ${ }^{7}$ Similar alterations in vascular tone in response to cold pressor testing could be demonstrated in patients with hypertension. ${ }^{8}$ Research indicates that the cold pressor test may be more sensitive for detection of abnormalities in the vascular endothelium than the more commonly used flow-mediated hyperemic response and may be less dependent on the effects of age and gender., 910 Both flow-mediated hyperemia and cold pressor testing can be used to assess the integrity of the vascular endothelium; the two methods may provide complementary information.

Thus, it was our intent for this protocol to assess the vascular effects of a newer combination estrogen/progestin postmenopausal HT agent and to do so using several complementary methods in order to definitively assess the responsiveness of the vascular endothelium.

\section{MATERIALS AND METHODS}

We recruited 18 postmenopausal (without menses for $\geq 12$ months) women with at least one traditional coronary heart disease (CHD) risk factor. The sample size of 18 women was estimated based on paired $t$ test to show a postulated 10\% change in flow-mediated brachial artery diameter during active treatment, compared with a $5 \%$ change during placebo therapy, with $90 \%$ power, an assumed standard deviation (SD) of $6 \%$, and about $10 \%$ dropout. These postulated changes were approximately based on the study performed by Gerhard et al. ${ }^{1}$ Change in brachial artery diameter during cold pressor testing was assessed as a secondary end point. Mean age of the study group was 62 years, and the range was 44-76 years. Study subjects were recruited from the VA Ann Arbor Women's Health Clinic, the University of Michigan clinics, or through community newspaper advertisement. Subjects were excluded if they had evidence of CHD, a history 
of venous thromboembolic disease, personal or family history of breast cancer, unexplained vaginal bleeding, recent ( $<3$ months) initiation of lipid-lowering therapy or use of hormone replacement therapy (HRT), and inability to give informed consent. The study protocol was approved by the University of Michigan Institutional Review Board as well as the VA Ann Arbor Human Studies Committee, and each subject gave written informed consent. All subjects underwent stress echocardiography to exclude significant $\mathrm{CHD}$ prior to randomization.

\section{Randomization to treatment}

The study was a double-blind, placebo-controlled, crossover trial. Each study participant was randomized to one of two sequences (groups) where one group received active treatment first $(n=9)$, and the other group received placebo first $(n=9)$. The therapeutic regimens lasted 3 months each, separated by a 1 -month washout period. Active treatment consisted of 10 $\mu \mathrm{g}$ ethinyl estradiol and $1 \mathrm{mg}$ norethindrone acetate given as a single tablet, taken once daily.

\section{Vascular reactivity studies}

Brachial artery studies were performed using two-dimensional ultrasound images according to established and validated methodology. Images were obtained using a $10-\mathrm{mHz}$ linear array transducer with a Phillips Image Point ultrasound system (HP, Andover, MA), used in conjunction with Vascular Tools (Medical Imaging Applications, Inc., Iowa City, IA) for ECG-triggered acquisition of image data sequences. Imaging was performed in the morning hours, after an overnight fast, with the participant resting supine for at least 10 minutes on a hospital bed in a quiet setting. Optimal brachial artery images were obtained between 2 and $10 \mathrm{~cm}$ above the antecubital crease. This location was marked, and all subsequent images were obtained at the same location. After baseline measurements, a blood pressure cuff was inflated to $200 \mathrm{~mm} \mathrm{Hg}$ over the proximal portion of the right arm for 4 minutes. Endothelial-dependent function was determined during the first 2 minutes of release of the cuff, as previous studies have shown that maximal dilatation occurs 1 minute after cuff deflation. The flow-dependent response was then allowed to return to baseline over a period of 10 minutes. Af- ter obtaining a second brachial artery baseline on the right arm, cold pressor testing was performed by immersing the left hand and forearm in ice slush for 2.5 minutes, with image acquisition on the right brachial artery occurring between 2 and 4 minutes. After another 10-minute resting period, endothelial-independent responsiveness was evaluated with $0.4 \mathrm{mg}$ nitroglycerin (NTG) administered sublingually. Brachial artery images were obtained 3 minutes after NTG administration. Four triggered events (defined as the end of the R wave on the ECG) for each intervention were recorded and acquired through a variable scan monochrome frame grabber for the PCI local bus (DT3152 Data Translation Inc, Marlboro, MA) attached to a Pentium II 400mHZ computer with 128MB SDRAM that allows for rapid and sophisticated video acquisition. Each triggered event consisted of 3 sequential frames, yielding a total of 12 analyzed images. The media/adventitia interface was chosen for both the near wall and the far wall of the artery. A linear portion of the vessel was chosen, and measurements from the 12 frames were averaged for every intervention. The end points of measurement were the percent change in diameter in response to reactive hyperemia, cold pressor testing, or NTG.

For repeat studies, images were recalled using a secondary monitor (Matrox, G200 Multi-Monitor, Quebec, Canada), and the images were aligned to ensure that precisely the same segment of the artery (at the same angle of ultrasound insonation) was imaged.

\section{Biochemical assays}

Blood was drawn on the day of brachial artery studies at pretreatment and at the completion of each treatment phase. Total cholesterol, low-density lipoprotein cholesterol (LDL-C), high-density lipoprotein cholesterol (HDLC), triglyceride, homocysteine, and high-sensitivity C-reactive protein (hsCRP) levels were sent to the University of Michigan clinical laboratory for immediate processing and analysis. Intercellular adhesion molecule-1 (ICAM-1), vascular cell adhesion molecule1 (VCAM-1), E-selectin, interleukin-6 (IL-6), and plasminogen activator inhibitor-1 (PAI-1) were measured using commercially available multiplexed biomarker immunoassay kits for Luminex instrumentation (Linco/Millipore, Billerica, MA). 


\section{Statistical analysis}

Descriptive statistics are reported as mean \pm SD for continuous variables and as proportions for categorical variables. Primary flow outcomes included flow-mediated hyperemic brachial artery response, cold pressor-mediated response, and NTG-mediated, endothelium-independent brachial artery response. Outcomes also included the biochemical assays, such as HDL-C, hsCRP, and VCAM. For each outcome, paired $t$ test was used to assess the unadjusted treatment effect. For each outcome, we also fit an additional regression model adjusting for potential period effect (effect that occurs in a given period irrespective of the sequence), carryover effect (treatment by period effect), and the respective pretreatment values of the outcome measure. In these analyses, we used the generalized estimating equation (GEE) model to adjust for correlation in outcomes measured at two different times within participants. ${ }^{11}$ The GEE model used measurements at two times as the dependent variable and active treatment indicator as the primary independent variable to detect the treatment effect along with other covariates, including baseline values of the outcome variable. All analyses were done using only participants with complete data and also using all available data, including those who dropped out. The GEE model was used for the latter analyses. All statistical analyses were done on the intent-to-treat basis, and a two-sided $p$ value was used to determine statistical significance of the treatment effect parameter estimate. We also assessed for correlations between biochemical assays and brachial artery responses by calculating pairwise correlations using Spearman's correlation coefficient.

\section{RESULTS}

Eighteen women were enrolled, underwent baseline brachial artery reactivity studies, had blood drawn for biochemical assays, and were randomized to one of two sequences: study drug first or placebo first per protocol. One participant experienced a lower extremity deep venous thrombosis (DVT) during active treatment and discontinued study participation. This participant was excluded from all analyses because no data were collected from her during any treatment phase of the study. Two other women dis- continued study participation for unrelated reasons (one sustained a back injury, and one developed a urinary tract infection), but during the second phase of the treatment sequence. Hence, all analyses were carried out on the 17 participants using all available data as well as the subset of 15 participants who completed the study. Baseline data, including CHD risk factors and medication use, are given in Table 1. In the study group, 1 woman had one CHD risk factor, 4 had two CHD risk factors, 6 had three risk factors, and 6 had four risk factors. Framingham risk scores for the women ranged from -2 to $17 ; 8$ women had scores of $\geq 10$.

Mean age of the 15 participants was 62 years (range 44-76 years). At pretreatment, study participants showed a mean increase in brachial artery diameter of $4.7 \pm 3.1 \%$ in response to hyperemic flow. Cold pressor testing induced a mean decrease in brachial artery diameter of $2.6 \pm 7.8 \%$, and NTG administration caused an increase in brachial artery diameter of $17.4 \pm$ $4.6 \%$ at pretreatment. We found no significant difference between placebo and active treatment in flow-mediated hyperemic brachial artery response $(6.2 \pm 4.7 \%$ vs. $4.7 \pm 4.8 \%, p=0.41)$, in cold pressor-mediated response $(3.3 \pm 8.3 \%$ vs. $0.4 \pm 8.3 \%, p=0.38)$ or in NTG-mediated, endothelium-independent brachial artery response $(15.8 \pm 7.1 \%$ vs. $18.7 \pm 8.7 \%, p=0.30)$. The results were similar with respect to the treatment

Table 1. Patient Characteristics of 17 Participants

\begin{tabular}{lc}
\hline Characteristic & Value $^{\mathrm{a}}$ \\
\hline Age, years & $62 \pm 10$ \\
Caucasian & $16(94 \%)$ \\
African American & $1(6 \%)$ \\
Hypercholesterolemia (TC $>200 \mathrm{mg} / \mathrm{dL}$, & $13(76 \%)$ \\
$\quad$ LDL $>130 \mathrm{mg} / \mathrm{dL}$, or current statin use) & \\
Hypertension & $10(59 \%)$ \\
Diabetes & $4(24 \%)$ \\
Family history of premature coronary & $14(82 \%)$ \\
$\quad$ disease & \\
Smoking & $10(59 \%)$ \\
Body mass index $\left(\mathrm{kg} / \mathrm{m}^{2}\right)$ & $27 \pm 7$ \\
Obese (BMI $\geq 30)$ & $6(35 \%)$ \\
Angiotensin-converting enzyme & $9(53 \%)$ \\
$\quad$ inhibitor use & \\
Beta-blocker use & $5(29 \%)$ \\
Calcium-channel blocker use & $2(12 \%)$ \\
Lipid-lowering therapy use & $5(29 \%)$ \\
\hline
\end{tabular}

aValues are mean \pm SD for continuous variables and number $(\%)$ of patients for discrete variables. 
Table 2. Mean Brachial Artery Responses $(n=15)^{\mathrm{a}}$

\begin{tabular}{|c|c|c|c|c|}
\hline & Pretreatment & Active treatment & Placebo & $\mathrm{p}$ value ${ }^{\mathrm{b}}$ \\
\hline Mean arterial diameter (mm) & $3.6 \pm 0.6$ & $3.6 \pm 0.5$ & $3.5 \pm 0.4$ & 0.07 \\
\hline$\% \Delta \mathrm{FMD}^{\mathrm{c}}$ & $4.7 \pm 3.1$ & $4.7 \pm 4.8$ & $6.2 \pm 4.7$ & 0.41 \\
\hline$\% \Delta \mathrm{CPT}$ & $-2.6^{*} \pm 7.8$ & $0.4 \pm 8.3$ & $3.3^{*} \pm 8.3$ & 0.38 \\
\hline$\% \Delta \mathrm{NTG}$ & $17.4 \pm 4.6$ & $18.7 \pm 8.7$ & $15.8 \pm 7.1$ & 0.30 \\
\hline
\end{tabular}

${ }^{a}$ Values are given as mean $\pm \mathrm{SD}$.

${ }^{b}$ From mean brachial artery response comparisons between active treatment and placebo. The $p$ values are similar when the comparisons are adjusted for period effect, carryover effect, and pretreatment values of the outcome measure or when adjusted for resting arterial diameter

c\% $\%$ FMD, diameter percent change from baseline during flow-mediated hyperemia; $\% \Delta \mathrm{CPT}$, diameter percent change from baseline during cold pressor testing; $\% \Delta \mathrm{NTG}$, diameter percent change from baseline during sublingual NTG administration.

${ }^{*} p$ value is 0.03 from comparing placebo and baseline $\% \Delta \mathrm{CPT}$.

effect size and the associated statistical significance when adjusted for potential period effect, carryover effect, and baseline values of the outcome measures using regression and when the analyses included data from all participants, including those who dropped out. Cold pressormediated brachial artery response improved significantly in the placebo phase compared with pretreatment responses, indicative of the wide variability of this response parameter. There was no evidence of phase or carryover effects in any of the outcome measures. A summary of brachial artery results is given in Table 2 .

Results of the biochemical assays are given for each phase of the study in Table 3. Significant de- crease in response to active treatment was seen for HDL-C, but only after adjustment for pretreatment value $(p=0.03)$. During active treatment, hsCRP significantly increased, whereas VCAM significantly decreased, and these results remained whether or not the comparisons were adjusted for their respective pretreatment values. Pairwise Spearman's correlation coefficient between biochemical assays and brachial artery responses showed a statistically significant correlation only between PAI-1 and flow-mediated brachial artery reactivity. For these parameters, there was a negative correlation seen in both the active treatment and the placebo phases of the study $(r=-0.56, p=0.03$ during active treat-

Table 3. Mean Biochemical Assay Responses $(n=15)^{\mathrm{a}}$

\begin{tabular}{lccrc}
\hline & Pretreatment & Active treatment & Placebo & p value $^{\mathrm{b}}$ \\
\hline TCholc $^{c}$ & $224 \pm 34$ & $215 \pm 40$ & $221 \pm 32$ & 0.30 \\
HDL-C & $60 \pm 17$ & $57 \pm 16$ & $57 \pm 11$ & 0.09 \\
LDL-C & $132 \pm 32$ & $127 \pm 36$ & $140 \pm 30$ & 0.07 \\
Triglycerides (mg/dL) & $151 \pm 77$ & $134 \pm 56$ & $124 \pm 43$ & 0.41 \\
Homocysteine & $9 \pm 3$ & $10 \pm 5$ & $10 \pm 3$ & 0.65 \\
hsCRP & $10 \pm 10$ & $15 \pm 8$ & $6 \pm 5$ & $<0.001$ \\
VCAM-1 & $1.2 \pm 0.5$ & $1.0 \pm 0.3$ & $1.3 \pm 0.3$ & 0.004 \\
ICAM-1 & $0.3 \pm 0.1$ & $27 \pm 10.1$ & $0.3 \pm 0.1$ & 0.42 \\
E-selectin (ng/mL) & $38 \pm 2$ & $4.7 \pm 4.4$ & $33 \pm 11$ & 0.08 \\
IL-6 & $4.3 \pm 3.1$ & $16 \pm 8$ & $4.9 \pm 4.0$ & 0.92 \\
PAI-1 & $21 \pm 14$ & & $17 \pm 9$ & 0.31 \\
\hline
\end{tabular}

a Values are given as mean \pm SD.

${ }^{b}$ From mean biochemical assay response comparisons between active treatment and placebo. The $p$ values are similar when the comparisons are adjusted for period effect, carryover effect, and pretreatment values of the outcome measure or when adjusted for resting arterial diameter

'TChol, total cholesterol (mg/dL); HDL-C, high-density lipoprotein (mg/dL); LDL-C, low-density lipoprotein $(\mathrm{mg} / \mathrm{dL})$; hs-CRP, high-sensitivity C-reactive protein $(\mu \mathrm{g} / \mathrm{mL})$; VCAM-1, vascular cell adhesion molecule-1 $(\mu \mathrm{g} / \mathrm{mL})$; ICAM-1, intercellular adhesion molecule-1 $(\mu \mathrm{g} / \mathrm{mL})$; IL-6, interleukin6 (pg/mL); PAI-1, plasminogen activator inhibitor-1 (ng/mL). 
ment; $r=-0.62, p=0.008$ during placebo). Thus, regardless of the presence of ethinyl estradiol/ norethindrone acetate in the subjects' bloodstream, increasing PAI-1 levels were negatively associated with brachial artery endotheliumdependent response.

\section{DISCUSSION}

Using ethinyl estradiol combined with northindrone acetate in a 3-month, placebo-controlled, crossover trial, we found no detectable effects on vascular reactivity of the brachial artery in postmenopausal women with risk factors for cardiovascular disease. Our findings contrast with the beneficial effects on the endothelium seen in some HT trials but support the lack of effect noted in other brachial artery studies, as well as in the Heart and Estrogen/progestin Replacement Study (HERS) and Women's Health Initiative (WHI) trials. ${ }^{1,3,4,12,13}$ We had postulated that the use of MPA was responsible for at least some of the adverse effects seen in randomized trials, as MPA has been shown in animal studies to diminish beneficial estrogen effects on the endothelium. ${ }^{5}$ Thus, we postulated that the use of norethindrone acetate as the progestin compound could have the potential to improve vascular endothelial function. Gerhard et al. ${ }^{1}$ studied the effects of a transdermal estrogen preparation combined with micronized vaginal progesterone in their crossover trial and found significant improvement in flow-mediated hyperemic responses to active therapy, both with estrogen alone and with estrogen plus progesterone. Their results may have differed from ours because they chose a transdermal estrogen preparation, which does not increase inflammatory markers, unlike oral estrogen preparations. ${ }^{14,15}$ They studied a population of postmenopausal hypercholesterolemic women of similar age to our study population, although a majority of our subjects had additional cardiovascular $(\mathrm{CV})$ risk factors as well. By contrast, Herrington et al. ${ }^{12}$ analyzed brachial artery flow-mediated responses in a large cohort population and found no significant difference in flow measurements between hormone users and nonusers in the subset of women who were older, had cardiovascular risk factors or disease, or used CV medications. Those women who were hormone users and were younger and had no risk factors, disease, or medications had significantly greater flow-mediated responses than corresponding hormone nonusers. ${ }^{12}$ In the Women's Angiographic Vitamin and Estrogen (WAVE) trial, a randomized controlled trial that looked at flow-mediated dilation (FMD) in response to $0.625 \mathrm{mg} /$ day conjugated equine estrogen (CEE) plus $2.5 \mathrm{mg}$ of MPA in postmenopausal women with established CHD, no changes in FMD were detected after 3 or 34 months. ${ }^{13}$

In our study, ethinyl estradiol plus norethindrone acetate treatment was associated with a significant increase in CRP levels and a modest, but significant, decrease in HDL levels. Both of these factors have been associated with adverse CV outcomes in larger trials and may explain the lack of positive endothelial effect seen in this study. Our finding that active treatment decreased HDL levels has been shown by other investigators studying the combination of oral estradiol with norethisterone acetate and is contrary to the usual HDL-raising effect of estrogen therapy alone. ${ }^{16}$ We did find a significant decrease in VCAM-1, an adhesion molecule that has been implicated in the cascade of atherogenesis and plaque instability when activated in the vascular endothelium. This has been shown by other groups in response to therapy with estrogen and estrogen alternatives. ${ }^{17,18}$

Our failure to find a significant effect for ethinyl estradiol/norethindrone acetate on vascular endothelial function may also be a consequence of the limitations of this method. Although there is a large body of literature indicating that brachial artery responses are predictive of CV outcomes, other investigators have found no correlation between CV effects and outcomes and brachial artery reactivity. ${ }^{19,20}$ Similarly, cold pressor testing has variable effects on vascular endothelium because of the variability with which individuals perceive it; in this study, we found it to be a poorly reproducible stimulus with wide variability in responses. It is possible that individuals may have become used to the iced slush effect after undergoing the test for the first time, although this is speculative, and we are not able to explain the high degree of variability we observed. Other investigators have reported that the cold pressor test induces inconsistent increases in plasma norepinephrine levels and that individuals with hypertension may 
have exaggerated pressor responses, whereas those prone to orthostatic hypotension may have an impaired response. ${ }^{21}$ Our finding of a significant correlation between PAI-1 levels and hyperemic, endothelium-dependent vascular reactivity may indicate that PAI-1, considered to be one of the most important determinants of how easily thrombi can be lysed in the arterial system, has important effects on vascular endothelial function as well.

In this older postmenopausal population with multiple CV risk factors, preexisting subclinical atherosclerosis is very probably present, and this fact may explain the lack of effect of ethinyl estra$\mathrm{diol} /$ norethindrone acetate more than any other factor. Estrogen receptor expression is markedly diminished in atherosclerotic arteries, ${ }^{22,23}$ and, thus, there may be no mechanism in aged, atherosclerotic vessels for estrogen to mediate a beneficial endothelial effect. Our results underscore negative findings in higher CHD risk women by other investigators, using multiple different HT preparations. ${ }^{12,13,24,25}$ The use of norethindrone acetate, a weakly androgenic synthetic progestin derived from nortestosterone, in this study has implications because of potential androgenic effects of this compound on vascular reactivity. However, exogenous androgen administration has not been shown to have any consistent effect on vascular reactivity. Some investigators have found that testosterone causes beneficial vasorelaxation, whereas others have demonstrated deleterious vasoconstrictor effects. ${ }^{26}$ As our patients had multiple, different risk factors, their responses to HT may have been differentially impaired by the differential contributions of these various risk factors and medication usage. Finally, the negative findings may have also resulted from our small sample size, given the highly variable hyperemic and cold pressor brachial responses shown in this and other studies. Although the results do not demonstrate even a trend toward an improvement, we cannot rule out the possibility that a larger study may uncover potentially beneficial vascular effects that were missed (type 2 error). Evidence does not suggest that this is a shortcoming of the methodology but rather the expected physiological variation of vascular studies. Larger follow-up studies, possibly comparing various different HT replacement strategies, are required to definitively resolve this issue.

\section{CONCLUSIONS}

Combined continuous ethinyl estradiol/norethindrone acetate does not improve brachial artery endothelial function in a small group of postmenopausal women at increased risk for $\mathrm{CV}$ disease. Whether this agent can induce beneficial effects on the vascular endothelium if studied in a larger group of women or in younger postmenopausal women without CV risk factors remains to be established.

\section{ACKNOWLEDGMENTS}

We thank Deborah Newman and Mary K. Medley for their administrative assistance and Allison Thodoroff and Kerri Briesmiester for their coordinator support.

\section{REFERENCES}

1. Gerhard M, Walsh B, Tawakol A, et al. Estradiol therapy combined with progesterone and endotheliumdependent vasodilation in postmenopausal women. Circulation 1998;98:1158.

2. Gilligan DM, Quyyumi AA, Cannon R. Effects of physiological levels of estrogen on coronary vasomotor function in postmenopausal women. Circulation 1994;89:2545.

3. Hulley S, Grady D, Bush T, et al. Randomized trial of estrogen plus progestin for secondary prevention of coronary heart disease in postmenopausal women. Heart and Estrogen/progestin Replacement Study (HERS) Research Group. JAMA 1998;280:605.

4. Rossouw JE, Anderson GL, Prentice RL, et al. Risks and benefits of estrogen plus progestin in healthy postmenopausal women: Principal results from the Women's Health Initiative randomized controlled trial. JAMA 2002;288:321.

5. Williams J, Honoré E, Washburn S, Clarkson T. Effects of hormone replacement therapy on reactivity of atherosclerotic coronary arteries in cynomolgus monkeys. J Am Coll Cardiol 1994;24:1757.

6. Anderson TJ, Uehata A, Gerhard MD, et al. Close relation of endothelial function in the human coronary and peripheral circulations. I Am Coll Cardiol 1995;26:1235.

7. Zeiher A, Drexler H, Wollschlaeger H, Saurbier B, Just J. Coronary vasomotion in response to sympathetic stimulation in humans: Importance of the functional integrity of the endothelium. I Am Coll Cardiol 1989;14:1181.

8. Antony I, Aptecar E, Lerebours G, Nitenberg A. Coronary artery constriction caused by the cold pressor 
test in human hypertension. Hypertension 1994;24: 212.

9. Corretti MC, Plotnick GD, Vogel RA. Correlation of cold pressor and flow-mediated brachial artery diameter responses with the presence of coronary artery disease. Am J of Cardiol 1995;75:783.

10. Corretti MC, Plotnick GD, Vogel RA. The effects of age and gender on brachial artery endothelium-dependent vasoactivity are stimulus-dependent. Clin Cardiol 1995; 18:471.

11. Liang K-Y, Zeger SL. Longitudinal data analysis using generalized linear models. Biometrika 1986;73:13.

12. Herrington DM, Espeland MA, Crouse JR 3rd, et al. Estrogen replacement and brachial artery flow-mediated vasodilation in older women. Arterioscl thromb Vascular Biol 2001;21:1955.

13. Kelemen M, Vaidya D, Waters DD, et al. Hormone therapy and antioxidant vitamins do not improve endothelial vasodilator function in postmenopausal women with established coronary artery disease: A substudy of the Women's Angiographic Vitamin and Estrogen (WAVE) trial. Atherosclerosis 2005;179:193.

14. Lacut K, Oger E, Le Gal G, et al. Differential effects of oral and transdermal postmenopausal estrogen replacement therapies on C-reactive protein. Thromb Haemost 2003;90:124.

15. Duvernoy C. Estrogen and C-reactive protein: Does an alternate route lead to a more attractive destination? Thromb Haemost 2003;90:1.

16. Zegura B, Guzic-Salobir B, Ebestjen M, Keber I. The effect of various menopausal hormone therapies on markers of inflammation, coagulation, fibrinolysis, lipids, and lipoproteins in healthy postmenopausal women. Menopause 2006;13:643.

17. Colacurci N, Manzella D, Fornaro F, Carbonella M, Paolisso G. Endothelial function and menopause: Effects of raloxifene administration. I Clin Endocrinol Metab 2003;88:2135.

18. Vitale C, Cornoldi A, Gebara O, et al. Interleukin-6 and flow-mediated dilatation as markers of increased vascular inflammation in women receiving hormone therapy. Menopause 2005;12:552.
19. Bottcher M, Madsen MM, Refsgaard J, et al. Peripheral flow response to transient arterial forearm occlusion does not reflect myocardial perfusion reserve. Circulation 2001;103:1109.

20. Stolen KQ, Kemppainen J, Kalliokoski KK, et al. Myocardial perfusion reserve and peripheral endothelial function in patients with idiopathic dilated cardiomyopathy. Am J Cardiol 2004;93:64.

21. Victor RG, Leimbach WN Jr., Seals DR, Wallin BG, Mark AL. Effects of the cold pressor test on muscle sympathetic nerve activity in humans. Hypertension 1987;9:429.

22. Losordo DW, Kearney M, Kim EA, Jekanowski J, Isner JM. Variable expression of the estrogen receptor in normal and atherosclerotic coronary arteries of premenopausal women. Circulation 1994;89:1501.

23. Post WS, Goldschmidt-Clermont PJ, Wilhide CC, et al. Methylation of the estrogen receptor gene is associated with aging and atherosclerosis in the cardiovascular system. Cardiovasc Res 1999;43:985.

24. Angerer P, Stork S, Kothny W, Schmitt P, von Schacky C. Effect of oral postmenopausal hormone replacement on progression of atherosclerosis: A randomized, controlled trial. Arterioscl, Thromb Vascular Biol 2001;21:262.

25. Byington RP, Furberg CD, Herrington DM, et al. Effect of estrogen plus progestin on progression of carotid atherosclerosis in postmenopausal women with heart disease: HERS B-mode substudy. Arterioscl, Thromb, Vascular Biol 2002;22:1692.

26. Wu FC, von Eckardstein A. Androgens and coronary artery disease. Endocr Rev 2003;24:183.

Address reprint requests to: Claire S. Duvernoy, M.D.

Veterans Affairs Ann Arbor Healthcare System University of Michigan Health System 2215 Fuller Road, IIIA Ann Arbor, MI 48105

E-mail: duvernoy@umich.edu 